\title{
A Survey on Various Models and Methods in Image Compression for an Efficient Image Retrieval
}

\author{
P. Arul Prabu \\ Research Scholar \\ Erode Arts \&Science College \\ Erode
}

\author{
S. Rizwana, $\mathrm{PhD}$ \\ Assistant Professor \& Head \\ Department of Computer Science \\ Erode Arts \&Science College \\ Erode
}

\begin{abstract}
Information technology dominates in every part of human survival in day to day's life. In earlier decades text processing were more popular due to the emergence in image processing which is user friendly and put the internal processing to a challengeable one. In order to overcome the challenges different methods have been proposed and the compression techniques marked a stable location in image processing. In this research, a deep survey is made on image compression its methodologies, algorithms etc. The study comprises on lossy compression, lossless compression and other hybrid compression techniques. Various image formats and their experimentation results were analysed along with its similarity measures such as compression ratio. This survey motivates to have a novel research in the field of image compression with more accuracy.
\end{abstract}

\section{Keywords}

Lossy compression, Lossless compression, MFOCPN, HAAR Wavelet, Debauchees Wavelet

\section{INTRODUCTION}

Due to the rapid development in the field of information technology various information processing system have been developed. In the initial days text processing dominates and due its various incompatibility images were used as input data. The main advantage of images processing can used by all class of people maybe a literate or illiterate. Digital image processing is a new methodology that processes a $2 \mathrm{D}$ image by a digital computer. In this process images with the various formats are been transformed into digital images for processing through computer systems. A digital image is represented in a matrix form with rows and columns with a finite number of bits. In image processing image storage become a challengeable due to the huge volume of data. Hence various methodologies have been proposed to overcome the storage issues.The basic image processing consist of various stages such as image acquisition, image enhancement, image segmentation, image compression, and image recognition and image storage. In digital image processing various areas are content based image retrieval, image segmentation, image compression, image mining etc. In general image processing takes place with various features low level features such as texture, edge, shape and colour. The main objective of image compression is to optimize the size of the image inorder to reduce the storage space of an image. The core concept behind this technique is to eliminate the redundant data. It is mainly used to reduce to decrease in bit or byte size of an image. Image Compression can be takes place mainly in two types. i. Lossy Compression and ii. Lossless Compressions. The reductionist approach is accomplished in three types of redundancy [1] as follows.
a. Coding redundancy
b. Inter pixel redundancy
c. Psycho visual redundancy

\section{LITERATURE REVIEW}

[1] To have an effective operations image compression is applied to reduce the file size and storage area. Traditional image compression methods and types provide the benefits related to these compression techniques. A hybrid image compression technique [2] inherits the characteristics of localizing the global spatial and frequency correlation from wavelets and classification and functional tasks from the modified forwardonly counter propagation neural network (MFOCPN) for image compression. Various benchmark test images are used to probe importance of the proposed technique. Another method for image compression [4] based on many methods that have been created for image compression which is called Five Modulus Method (FMM). It converts each pixel value in an $8 \times 8$ block into a multiple of 5 for each of the R, G and B arrays. Then, the new values could be divided by 5 to get 6-bit length values for each pixel which is lesser than in storage space than the original value which is 8-bits. One more techniques for compression by the new values as a stream of bits has been presented that give the space to store and transfer the resultant image [5].

Various applications[7] of image processing such as satellite imaging, medical diagnosis and multimedia inputs holds huge size and requires large amount of storage space or high bandwidth for transmission in its original form and image compression techniques can be used many applications. Lossless image compression techniques preserve the information. A lossy method [8] to compress a digital image by addition method for image compression. The image is quantized into set of blocks of size $4 \times 4$. A nonlinear geometric transform, called peak transform (PT) [9], is applied for efficient image representation and coding. The Peak Transform is able to convert highfrequency signals into low-frequency ones, making them much easier to be compressed. With the combined wavelet transform and sub-band decomposition, this method is able to reduce the signal energy in HF sub-bands and target a significant transform coding gain.

The Discrete wavelet Transform (DWT) [10] with EZW and SPIHT algorithms is used for an efficient compression.DWT reduces the noise levels and it is useful in its high speed and accuracy. EZW and SPIHT algorithms are based on lossy compression and the bit rate determines its accuracy. Image compression [11], image coder is applied in various types of image compression techniques and the main objective is to decrease the redundancy of the image data which helps in increasing the capacity of storage and efficient transmission [12] Generally, a compression method helps in decreasing the size of an image with disturbing the quality of the image. The utilisation 
of neural-network for bi-level image compression [13]. In this lossy compression method, the locations of pixels of image inputted to a multilayer perceptron neural-network. The final weights of the trained neural-network are quantised, represented by a few bits, Huffman encoded and then the output image is stored as the compressed image.

A Lossless compression technique assures the reconstruction of the original information without incurring any distortion in the process [14].The performance of several lossless gray scale image compression algorithms like CALIC is applied for performance evaluation. Another technique of Image processing is applied by considering Haar wavelet [15] and Vector transform techniques. Higher compression rate is achieved with Integer-to-Integer transform and Band-let image compression, low SNR Signal to Noise Ratio) values and high RMSE values.

Complex image compression is accomplished on the basis of lossy and lossless compression. Lossy compression is a data encoding method that compresses data by losing data during the process. Lossless compression is used to compress the image without any data loss of data [16].

Another method [17] consists of seven operations which are the sampling, the partitioning, the transform, the quantization, the entropy coding and Huffman coding. Various techniques are been analysed [18] and provided the basics of image coding with a discussion of vector quantization and wavelet compression under vector quantization. It identifies the advantageous features and helps in selecting ultimate method for compression. [19] Communication media is image compression. Compression makes it possible for creating file sizes of manageable, storable and transmittable dimensions. Many techniques [20] to reduce the compression ratio, and increase usability of fast computation with certain limits, there are lots of innovative ways that are yet to overcome these limits. Due to reserved bandwidth and capacity, images need be compressed and soft encoded before further using it in transmission process. It points out Lossy as well as Lossless compression techniques as they are used in fields of image processing

\section{METHODOLOGY}

Compression techniques are divided into two types: lossless and lossy. When lossless data is decompressed, the resultant image is same as original. Lossy compression techniques result in loss of data and the decompressed image is not accurately the identical of the original.[24]

\subsection{Lossless Compression}

In lossless compression technique the reconstructed image, after compression, is identical to the original image. It can be applied in many applications such as file formatting \& UNIX tool zip. It is important when the input $\&$ the resultant data be uniform. The file formats like PNG or GIF are lossless compression. Most lossless compression take place as generates a statistical model for the input data, and uses this model to map input data to bit sequences in such a way that "probable" data will produce shorter output than "improbable" data.

\subsection{Lossy Compression}

Lossy compression techniques provides superior compression ratio than lossless compression. The compression ratio is high; the decompressed image is not uniform to the original image, but close to it. Different types of lossy compression techniques are widely used; it is characterized by the quality of the reconstructed images and its adequacy for application.[9]

\subsection{Classical wavelet based method with MFO-CPN[2]}

This method MFO-CPN passed the significant wavelet coefficients instead of passing the whole image after applying wavelet transform to Image. It gives a better compression rate because is achieved by wavelet transform and has compression with MFO-CPN in next levels.

The wavelet coefficients are quantized, and encoded without any loss by the entropy encoder box, which usually employs contextual information. Top level compression are obtained by increasing the quantization step and by making better anticipation for the ranges of quantized values via appropriate contexts and data structures.

The MFOCPN is applied for predicting wavelet coefficients, and training is accomplished for each wavelet level and sub band with threshold. It is uses in the way that lower sub bands of wavelets has significant data and required important information, In Figure 1, a typical wavelet sub band decomposition is represented. The notation $\mathrm{L}$ and $\mathrm{H}$ stand respectively for low pass and high pass and the $\mathrm{LH}, \mathrm{HL}$, and $\mathrm{HH}$ coefficients are theresiduals computed from these predictors. The MFOCPNbasically acts as the function approximate and predicts the useful coefficients.

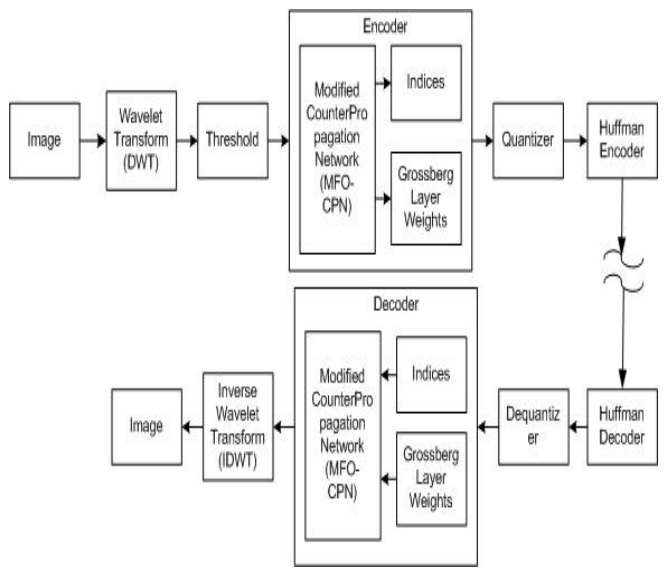

Fig:1 wavelet sub band decomposition

It requires minimum number of clusters to hold these data and store all the necessary wavelet coefficients in these clusters indices and Grossberg layer weights. The cluster indices and Grossberg layer weights which require less number of bits and we can achieve higher compression ratio.

\subsection{Wavelet packet best tree based on Threshold Entropy [3]}

It is a tree based on Threshold Entropy technique on wavelet packet with lossy enhanced run-length encoding. Selection of the sub-bands, which and reduction in the time complexity of wavelet packets decomposition, The Threshold entropy criterion finds the information contains of transform coefficients of subbands. The information contains of decomposed components of wavelet packets may be greater than or less than the information contain of component, which has been decomposed. The Threshold entropy of child nodes is checked withcost of component, which has been decomposed. When the sum of the cost of child nodes is lesser than parent node, then the child nodes are considered as leaf nodes of the tree, otherwise child nodes are removed from the tree, and parent node becomes leaf node of the tree. This process is iterated up to the last level of decomposition. 


\subsubsection{Algorithm Entropy}

1. Select the image

2. Initialize the current node equal to input image

3. Decompose the current node using wavelet packet tree

4. Estimate the cost of current node, and decomposed components

5. Compare the cost of parent node with child nodes. When the sum of cost of child nodes is greater than

the parent node, consider the parent node as leaf node of the tree, and child nodes are pruned, else repeat the steps 3, 4, and 5 for each child node by considering a child node as a current node, until last level of the tree reached.

\subsection{Enhanced Run-Length Encoding}

Enhanced Run-Length Encoding is well known Huffman coding or Arithmetic coding methods. The issues with existing Run Length Encoding, is that the compression ratio obtained from run-length encoding schemes vary based on the data to be encoded with redundancy. In the Enhanced RLE, the neighbouring coefficients are compared, with acceptable value, which is provided by the user according to the applications.

\subsection{Traditional Wavelets [6]}

3.6.1 Haar wavelet: Haar used functions to give a countable orthonormal system for the space of square integrable functions on the real line. As a special case of the Daubechies wavelet, it is also known as D2. The Haar wavelet is also the simplest possible wavelet. The processing demerits of the Haar wavelet are that it is not continuous, and therefore not differentiable. This property can, however, be an advantage for the analysis of signals with sudden transitions, such as monitoring of tool failure in machines.

3.6.2 Daubechie wavelet: Daubechies wavelets are chosen to have the highest number A of vanishing moments, for given support width $\mathrm{N}=2 \mathrm{~A}$, and among the $2 \mathrm{~A}-1$ possible solutions the one is chosen whose scaling filter has external phase. The wavelet transform is also easy to put into practice using the fast wavelet transform. Daubechies wavelets are widely applied in a broad range of problems, such as self-similarity properties of a signal or fractal problems, signal discontinuities, etc.

3.6.3 Biorthogonal Wavelet: Bi-Orthogonal wavelets belong to the families of orthogonal wavelets and a well-known fact in the filter theory community that symmetry and perfect reconstruction are incompatible (except for the Haar wavelet) when the same FIR filters are used for decomposition and for reconstruction process.

3.6.4 Coiflet wavelet: Coiflets are discrete wavelets designed by Ingrid Daubechies with scaling functions with vanishing moments. The wavelet is near symmetric, their wavelet functions have $N / 3$ vanishing moments and scaling functions $N$ / $3-1$, and has been used in many applications using CalderonZygmund Operators. Both the scaling function (low-pass filter) and the wavelet function (High-Pass Filter) must be normalised by a factor

3.6.5 Symlet wavelet: Symlets constitute a family of almost symmetric wavelets proposed by Daubechies by modifying the construction of the $d b N$. The other properties of the two families are similar. Symlets of orders 2 to 8 (syml is simply the Haarwavelet). The idea of construction consists of re-using the $m 0$ function introduced for $d b N$, considering $|\mathrm{m} 0(\mathrm{w})| 2$ as a function $W$ of the variable $z=e i w$. It an factorize $W$ in various manners in the form of $W(z)=U(z) U\left(z^{-1}\right)$, since the roots of $W$ with module different from 1 go inpairs: if $z l$ is a root then $z l-1$ is also a root. By constructing $U$ so that its roots are all of module $<1$ we construct the Daubechies wavelets $d b N$. The filter $U$ has a minimal phase.

\subsubsection{D-DCT BASED TECHNIQUES[22]}

3D-DCT has been used in many techniques.3D-DCT is frequently used to represent the object appearance model that is robust to variations in illumination,pose,etc.Furthermore,3DDCT is used in many image processing applications, such as video watermarking, denoising , and fingerprinting 3D-DCT sequential coding is used for specific classes of images like medical images. In 3Dspiral JPEG image compression is used, where it uses spiral scanning to format the multi-dimensional constellation, in order to get a more effective compression scheme.In videocompression,3D-DCT is used by taking the twodimensional frame sand the temporal dimension(the sequence of frames) as the basis.

\section{PERFORMANCE EVALUATION 4.1.Compression Ratio}

Compression ratio is formulated as the ratio of bits uncompressed to bits compressed.

$$
\text { Compression Ratio }=\frac{\text { Uncompressed Size }}{\text { Compressed Size }}
$$

\section{CONCLUSION}

Due to the rapid development in the field of digital image processing, especially in the field of Image compression, it is necessary to analyse the compression methodologies to propose a novel methodology. In this paper a deep study on various image compression methods have been analysed and their methods are observed. Also the study takes place from the initial methodology i.e. lossy and lossless compression to the advanced image compression techniques. Compared to the existing studies, it provides better knowledge to travel in an advanced path of image compression.

\section{REFERENCES}

[1] D.Meenakshi and V.Karthika Devi, "LITERATUR REVIEW OF IMAGE COMPRESSIONTECHNIQUE" ,International Journal of Computer Science \& Engineering Technology(IJCSET), Vol.6, pp.286-288, ISSN : 22293345,May 2015

[2] AshutoshDwivedi et al., "A Novel Hybrid Image Compression Technique: Wavelet-MFOCPN", Proc. of ASID '06, 8-12 Oct,pp.492-495.

[3] Prof. Dr. G. K. Kharate and Dr.Mrs. V. H. Patil,"Color Image Compression Based On Wavelet PacketBest Tree", International Journal of Computer Science Issues, Vol. 7,Issue 2, No 3,pp.31-35, March 2010

[4] Firas A. Jassim , Hind E. Qassim," FIVE MODULUS METHOD FOR IMAGE Compression, pp.19-28, October 2012

[5] GauravVijayvargiya et al., “ A Survey: VariousTechniques of Image Compression", International Journal of Computer Science and Information Security, Vol. 11, No. 10, October 2013

[6] Dr.BEswara Reddy1 and K VenkataNarayana," A LOSSLESS IMAGE COMPRESSION USING TRADITIONAL AND LIFTING BASED WAVELETS", 
An International Journal(SIPIJ) Vol.3, No.2,pp.213-222, April 2012

[7] Malkitsingh, REVIEW OF LOSSLESS IMAGECOMPRESSION, International Journal of Scientific Research Engineering \& Technology (IJSRET), ISSN2278 - 0882Volume 5,Issue 2,pp.53-55, February 2016.

[8] Nadeem Akhtar1, Salman Khan2, Gufran Siddiqui3," A Novel Lossy Image Compression Method”, 2014 Fourth International Conference on Communication Systems and Network Technologies, pp.866-870, 978-1-4799-3070-8/14 $\$ 31.00$ @ 2014 IEEE

[9] S.Anila and Dr.N.Devarajan, AN EFFICIENT IMAGE COMPRESSIONTECHNIQUE USINGPEAKTRANSFORM, 2009 International Conference on Computer Technology and Development,pp.395-399, 978-0-7695-3892-1/09 $\$ 26.00$ @ 2009 IEEE

[10] Yasaswi Velamuri1, Sandhya Patnayakuni2, Nancharaiah Vejendla3, Image Compression Based On The TransformDomain Downward Conversion ,InternationalJournal of Engineering Research and Development, Volume 9, Issue 12 (February 2014), PP. 1320 pp.206-210,e-ISSN: 2278-067X, p-ISSN: 2278-800X,

[11] Dr.Ajit Singh*, MeenakshiGahlawat,'Image Compression and its Various Techniques, International Journal of Advanced Research in Computer Science and Software Engineering,Volume 3, Issue 6, June 2013,pp.650654,ISSN: $2277128 \mathrm{X}$

[12] Malwinder Kaur 1, Navdeep Kaur 2, A LITREATURE SURVEY ON LOSSLESS IMAGECOMPRESSION, International Journal of Advanced Research in Computer andCommunication Engineering, Vol. 4, Issue 3, March 2015, ISSN (Online) 2278-1021, ISSN (Print) 23195940

[13] S. Sahami1 M.G. Shayesteh, Bi-level image compression technique usingneuralnetworks", Vol. 6, Iss. 5, pp. 496506, The Institution of Engineering and Technology2012 IET Image Processing, 10.1049/iet-ipr.2011.0079

[14] Miss. Rohini N. Shrikhande, IMAGE COMPRESSION USING CALIC, 2014 International Conference on Advances in Communication and Computing Technologies, 978-1-4799,7319-4/14/\$31.00 @2014 IEEE, ICACACT 2014
[15] NehaSikka and Sanjay Singla, Lossless Image Compression Technique using HaarWavelet and Vector Transform, International Conference on Research Advances inIntegrated navigation Systems (RAINS-2016),April 0607, 978-1-4673-8819-8,IEEE

[16] Dr. M. Sundaresan, E. Devika, Image Compression Using H.264 And DeflateAlgorithm,Proceedings of the International Conference on Pattern Recognition, Informatics and Medical Engineering, March 21-23, 2012, 978-1-4673-1039-0/12/\$31.00 @2012 IEEE

[17] QusayKanaanKadhim,'Image Compression Using Discrete Cosine Transform Method",International Journal of Computer Science and Mobile Computing,IJCSMC,Vol. 5, Issue. 9, September 2016, pg.186 - 192,ISSN 2320-088X

[18] G.M.Padmaja, 2 P.Nirupama, Analysis of Various Image Compression Techniques, ARPN Journal of Science and Technology, VOL. 2, NO. 4,pp.371-376, May 2012, ISSN 2225-7217

[19] SonalChawla, 2Meenakshi Beri, 3Ritu Mudgil, Image Compression Techniques: A Review,International Journal of Computer Science and Mobile Computing, Vol.3 Issue.8, August- 2014, pg. 291-296 ISSN 2320-088X

[20] Dinesh V. Rojatkar, Nitesh D. Borkar, Buddhabhushan R. Naik, Ravindra N. Peddiwar, Image Compression Techniques: Lossy and Los, International Journal of Engineering Research and General Science Volume 3, Issue 2,pp.912-917March-April, 2015, ISSN 2091-2730

[21] Surabhi N, 2 Sreeleja N Unnithan, Image Compression Techniques:A Review, International Journal of Engineering Development and ResearchVolume5 , Issue1,pp.585589,|ISSN:2321-9939

[22] Mohamed E. Emara, Rehab F. Abdel-Kader, and Mohamed S. Yasein, ImageCompression Using Advanced Optimization Algorithms, Journa lof CommunicationsVol.12,No.5, pp.271-278, May2017

[23] Rahul Sood, Vikramjit Singh Bhathal, Sukhchain Singh,Image Compression Techniques: A Review,International Research Journal of Engineering and Technology (IRJET),Volume: 05 Issue: 04 ,pp.3855-3856,| Apr-2018,e-ISSN: $\quad$ 2395-0056p-ISSN: 23950072(www.cs.cf.ac.uk/Dave/Multimedia/node210.html). 